Supporting information for:

\title{
Quantifying precision loss in targeted metabolomics based on mass spectrometry and nonmatching internal standards
}

Authors: Arve Ulvik, Adrian McCann, Øivind Midttun, Klaus Meyer, Keith M Godfrey ${ }^{1}$, Per M Ueland

Bevital, laboratoriebygget, 9 etg. Jonas Lies veg 87, 5021 Bergen, Norway

${ }^{1}$ MRC Lifecourse Epidemiology Unit and NIHR Southampton Biomedical Research Centre, University of Southampton and University

Hospital Southampton NHS Foundation Trust

List of contents:

Table S1. CVs for analytes and internal standards based on uncorrected peak areas on platform GC

Table S2. CVs for analytes and internal standards based on uncorrected peak areas on platform LC1

Table S3. CVs for analytes and internal standards based on uncorrected peak areas on platform LC2

Table S4. CVs for analytes and internal standards based on uncorrected peak areas on platform LC3

Table S5. CVs for matching and nonmatching A/IS on platform GC

Table S6. CVs for matching and nonmatching A/IS on platform LC1

Table S7. CVs for matching and nonmatching A/IS on platform LC2

Table S8. CVs for matching and nonmatching A/IS on platform LC3 
Table S1. CVs for analytes and internal standards based on uncorrected peak areas on platform GC

\begin{tabular}{|c|c|c|c|c|c|c|}
\hline \multirow[b]{3}{*}{ Analyte } & \multirow[b]{3}{*}{$\mathrm{IS}^{\mathrm{a}}$} & \multirow[b]{3}{*}{ conc. $(\mu \mathrm{M})^{\mathrm{b}}$} & \multicolumn{4}{|c|}{ CV $(\%)$} \\
\hline & & & \multicolumn{2}{|c|}{ within-run ${ }^{c}$} & \multicolumn{2}{|c|}{ between-run } \\
\hline & & & analyte & internal standard & analyte & internal standard \\
\hline methylmalonic acid & ${ }^{2} \mathrm{H}_{3}$ & 1.2 & $6.8(3.9,31)$ & $6.7(3.8,34)$ & 8.7 & 8.7 \\
\hline 2-Hydroxybutyrate & ${ }^{2} \mathrm{H}_{3}$ & 50 & $6.3(3.2,103)$ & $5.8(2.4,103)$ & 12.3 & 12.0 \\
\hline 3-Hydroxybutyrate & ${ }^{2} \mathrm{H}_{2}$ & 70 & $5.9(3.2,105)$ & $6.1(3.4,105)$ & 11.9 & 12.1 \\
\hline alanine & ${ }^{2} \mathrm{H}_{3}$ & 182 & $5.4(2.7,26)$ & $5.4(2.7,27)$ & 10.3 & 10.2 \\
\hline 3-Hydroxyisobutyrate & ${ }^{2} \mathrm{H}_{5}$ & 32 & $6.6(3.8,103)$ & $5.9(3.4,104)$ & 12.3 & 11.7 \\
\hline glycine & ${ }^{2} \mathrm{H}_{2}$ & 90 & $5.5(1.8,101)$ & $5.1(2.2,99)$ & 11.0 & 10.9 \\
\hline sarcosine & ${ }^{2} \mathrm{H}_{3}$ & 2.7 & $6.1(2.8,24)$ & $5.8(2.3,25)$ & 8.0 & 7.9 \\
\hline valine & ${ }^{2} \mathrm{H}_{2}$ & 9.5 & $5.2(1.9,99)$ & $6.1(2.6,98)$ & 11.9 & 12.7 \\
\hline leucine & ${ }^{2} \mathrm{H}_{10}$ & 50 & $6.0(3.5,104)$ & $5.2(2.3,106)$ & 12.2 & 19.3 \\
\hline isoleucine & ${ }^{2} \mathrm{H}_{10}$ & 61 & 6.2 (3.3 32) & $6.0(3.2,32)$ & 8.5 & 8.3 \\
\hline a-Ketoglutarate & ${ }^{2} \mathrm{H}_{6}$ & 64 & $6.9(2.9,34)$ & $7.2(3.8,34)$ & 9.4 & 9.6 \\
\hline threonine & ${ }^{2} \mathrm{H}_{2}$ & 87 & $6.6(3.3,25)$ & $5.8(2.4,28)$ & 9.5 & 8.6 \\
\hline proline & ${ }^{2} \mathrm{H}_{3}$ & 48 & $5.2(2.3,100)$ & $5.3(2.5,101)$ & 11.1 & 11.2 \\
\hline glutamine & ${ }^{2} \mathrm{H}_{5}$ & 239 & $9.0(3.2,42)$ & $9.0(3.9,42)$ & 11.6 & 11.7 \\
\hline serine & ${ }^{2} \mathrm{H}_{3}$ & 68 & $5.1(2.2,26)$ & $5.2(1.7,27)$ & 7.1 & 7.1 \\
\hline methionine & ${ }^{2} \mathrm{H}_{4}$ & 23 & $5.6(3.4,24)$ & $5,7(2.8,24)$ & 9.2 & 8.4 \\
\hline
\end{tabular}




\begin{tabular}{|c|c|c|c|c|c|c|}
\hline glutamic acid & ${ }^{2} \mathrm{H}_{5}$ & 32 & $8.2(3.8,28)$ & $8.9(5.0,27)$ & 10.7 & 11.1 \\
\hline phenylalanine & ${ }^{2} \mathrm{H}_{8}$ & 31 & $5.2(1.7,34)$ & $5.0(2.133 .4)$ & 8.3 & 8.1 \\
\hline homocysteine & ${ }^{2} \mathrm{H}_{4}$ & 30 & $5.0(2.0,26)$ & $5.5(2.625 .4)$ & 8.0 & 7.7 \\
\hline ornithine & ${ }^{2} \mathrm{H}_{3}$ & 42 & $7.9(3.2,31)$ & $7.6(3.6,30)$ & 10.7 & 10.3 \\
\hline lysine & ${ }^{2} \mathrm{H}_{9}$ & 40 & $7.3(3.0,31)$ & $7.6(3.3,32)$ & 9.2 & 9.6 \\
\hline tyrosine & ${ }^{2} \mathrm{H}_{2}$ & 230 & $6.0(2.6,28)$ & $5.8(2.7,29)$ & 7.7 & 7.7 \\
\hline tryptophan & ${ }^{2} \mathrm{H}_{5}$ & 36 & $12.1(4.3,31)$ & $13.1(4.7,35)$ & 14.1 & 15.6 \\
\hline cystathionine & ${ }^{2} \mathrm{H}_{4}$ & 2.3 & $13.7(4.8,35)$ & $13.8(4.2,35)$ & 16.9 & 16.5 \\
\hline kynurenine & ${ }^{2} \mathrm{H}_{3}$ & 1.8 & $15.0(4.4,75)$ & $14.0(4.476)$ & 19.0 & 18.6 \\
\hline
\end{tabular}

${ }^{a}$ Number and type of stable isotope substitutions for internal standard. ${ }^{b}$ concentration of internal standard. ${ }^{c}$ median (range) across 60 runs. Abbreviations IS, internal standard. 
Table S2. CVs for analytes and internal standards based on uncorrected peak areas on platform LC1

\begin{tabular}{|c|c|c|c|c|c|c|}
\hline \multirow[b]{3}{*}{ Analyte } & \multirow[b]{3}{*}{$\mathrm{IS}^{\mathrm{a}}$} & \multirow[b]{3}{*}{ conc. $(\mu \mathrm{M})^{\mathrm{b}}$} & \multicolumn{4}{|c|}{ CV (\%) } \\
\hline & & & \multicolumn{2}{|c|}{ within-run ${ }^{c}$} & \multicolumn{2}{|c|}{ between-run } \\
\hline & & & analyte & internal standard & analyte & internal standard \\
\hline histidine & ${ }^{2} \mathrm{H}_{3}$ & 66 & $3.6(1.9,18.5)$ & $3.8(2.0,16.4)$ & 6.0 & 6.3 \\
\hline arginine & ${ }^{2} \mathrm{H}_{3}$ & 30 & $3.2(1.8,33.3)$ & $3.3(1.6,33)$ & 8.4 & 8.4 \\
\hline dimethylglycine & ${ }^{2} \mathrm{H}_{2}$ & 5 & $6.0(3.4,118)$ & $3.9(2.0,114)$ & 22.5 & 22 \\
\hline 1-methylhistidine & ${ }^{2} \mathrm{H}_{3}$ & 20 & $4.9(2.3,19.3)$ & $4.4(2.2,19.3)$ & 7.0 & 6.7 \\
\hline methionine sulfoxide & ${ }^{2} \mathrm{H}_{5}$ & 5 & $8.4(4.6,28)$ & $7.2(3.9,24)$ & 11.6 & 9.5 \\
\hline trimethyllysine & ${ }^{2} \mathrm{H}_{2}$ & 90 & $4.8(2.5,27)$ & $4.1(2.1,25)$ & 8.4 & 7.3 \\
\hline creatine & ${ }^{2} \mathrm{H}_{3}$ & 50.6 & $5.3(3.128)$ & $5.5(2.3,25)$ & 9.0 & 8.3 \\
\hline betaine & ${ }^{2} \mathrm{H}_{2}$ & 20 & $3.9(1.9,63)$ & $3.3(1.6,52)$ & 14.5 & 12.3 \\
\hline homoarginine & ${ }^{2} \mathrm{H}_{10}$ & 1.25 & $7.5(3.8,19.1)$ & $7.3(3.6,21)$ & 9.2 & 8.9 \\
\hline trimethylamine $\mathrm{N}$-oxide & ${ }^{2} \mathrm{H}_{10}$ & 35.6 & $4.4(2.8,31)$ & $3.6(1.4,29)$ & 9.0 & 8.2 \\
\hline 3-methylhistidine & ${ }^{2} \mathrm{H}_{6}$ & 14.8 & $7.1(4.0,47)$ & $6.3(2.9,41)$ & 13.2 & 12.0 \\
\hline choline & ${ }^{2} \mathrm{H}_{2}$ & 10 & $3.6(1.7,60)$ & $3.3(1.9,67)$ & 14.5 & 15.5 \\
\hline symmetric dimethylarginine & ${ }^{2} \mathrm{H}_{3}$ & 2 & $11.6(6.5,30)$ & $12.6(3.7,45)$ & 14.8 & 16.5 \\
\hline asymmetric dimethylarginine & ${ }^{2} \mathrm{H}_{5}$ & 1 & $10.5(3.7,20)$ & $10.3(4.8,19.5)$ & 12.1 & 12.0 \\
\hline methionine & ${ }^{2} \mathrm{H}_{3}$ & 70 & $4.1(2.3,23)$ & $4.0(1.7,16.9)$ & 5.1 & 4.9 \\
\hline creatinine & ${ }^{2} \mathrm{H}_{4}$ & 94 & $4.0(1.3,9.9)$ & $3.8(0.7,283)$ & 4.4 & 19.4 \\
\hline
\end{tabular}


${ }^{a}$ Number and type of stable isotope substitutions for internal standard. ${ }^{b}$ concentration of internal standard. ${ }^{\mathrm{C}}$ median (range) across 60 runs. Abbreviations IS, internal standard. 
Table S3. CVs for analytes and internal standards based on uncorrected peak areas on platform LC2

$\mathrm{CV}(\%)$

\begin{tabular}{|c|c|c|c|c|c|c|}
\hline & & & & & & \\
\hline & & & wit & run $^{\mathrm{c}}$ & & n-run \\
\hline Analyte & $I^{a}$ & conc. $(\mathrm{nM})^{\mathrm{b}}$ & analyte & internal standard & analyte & internal standard \\
\hline pyridoxal 5'-phosphate & ${ }^{2} \mathrm{H}_{2}$ & 100 & $5.7(2.6,23)$ & $5.4(1.5,18.7)$ & 10.3 & 8.8 \\
\hline neopterin & ${ }^{15} \mathrm{~N}_{1}$ & 25 & $6.4(3.3,19.9)$ & $6.1(3.2,18.8)$ & 8.7 & 8.3 \\
\hline cystathionine & ${ }^{2} \mathrm{H}_{4}$ & 200 & $6.1(2.6,15.4)$ & $6.1(2.1,16.0)$ & 8.5 & 8.6 \\
\hline trigonelline & ${ }^{2} \mathrm{H}_{3}$ & 1000 & $4.1(2.2,14.5)$ & $4.4(1.4,15.3)$ & 6.6 & 6.7 \\
\hline thiamine monophosphate & ${ }^{2} \mathrm{H}_{3}$ & 10 & $7.6(3.3,21)$ & $6.6(3.4,19.2)$ & 12.2 & 8.1 \\
\hline trimethylamine $\mathrm{N}$-oxide & ${ }^{2} \mathrm{H}_{9}$ & 10 & $5.3(2.0,21)$ & $5.9(1.5,22)$ & 7.6 & 8.6 \\
\hline $1 \mathrm{~N}$-methylnicotinamide & ${ }^{2} \mathrm{H}_{4}$ & 250 & $5.5(2.2,21)$ & $5.9(1.5,22$ & 9.7 & 8.6 \\
\hline nicotinic acid & ${ }^{2} \mathrm{H}_{4}$ & 100 & $6.7(3.3,20)$ & $6.1(2.3,22)$ & 9.7 & 8.7 \\
\hline trans-3'-hydroxycotinine & ${ }^{2} \mathrm{H}_{3}$ & 100 & $6.3(1.9,27)$ & $6.6(2.6,30)$ & 9.4 & 10.3 \\
\hline nicotinamide & ${ }^{2} \mathrm{H}_{4}$ & 250 & $7.4(2.4,27)$ & $6.6(2.9,29)$ & 11.1 & 9.7 \\
\hline 4-pyridoxic acid & ${ }^{2} \mathrm{H}_{2}$ & 100 & $4.9(1.9,19.2)$ & $5.1(2.519 .8)$ & 7.1 & 8.3 \\
\hline pyridoxal & ${ }^{2} \mathrm{H}_{3}$ & 25 & $5.1(2.7,20)$ & $4.7(1.7,24)$ & 7.2 & 7.3 \\
\hline flavin mononucleotide & ${ }^{2} \mathrm{H}_{7}$ & 30 & $6.1(2.6,22)$ & $5.1(1.2,14.8)$ & 9.1 & 6.4 \\
\hline pyridoxin & ${ }^{2} \mathrm{H}_{4}$ & 20 & $4.3(1.5,17)$ & $5.5(1.7,19.4)$ & 6.3 & 6.9 \\
\hline cotinine & ${ }^{2} \mathrm{H}_{3}$ & 200 & $4.3(1.4,16.1)$ & $5.0(1.5,19.0)$ & 6.2 & 6.7 \\
\hline 3-hydroxykynurenine & ${ }^{2} \mathrm{H}_{2}$ & 50 & $4.3(1.4,18.2)$ & $4.8(1.4,18.0)$ & 7.3 & 6.7 \\
\hline
\end{tabular}




$\begin{array}{lcccccc}\text { 3-hydroxyanthranilic acid } & { }^{2} \mathrm{H}_{2} & 50 & 6.1(2.3,27) & 4.7(1.6,18.7) & 8.8 & 8.1 \\ \text { xanthurenic acid } & { }^{2} \mathrm{H}_{4} & 30 & 5.9(1.7,17.6) & 5.8(1.6,24) & 8.2 & 7.1 \\ \text { riboflavin } & { }^{2} \mathrm{H}_{8} & 30 & 5.7(2.7,17.6) & 5.8(3.0,19.1) & 7.4 \\ \text { kynurenic acid } & { }^{2} \mathrm{H}_{5} & 100 & 4.8(1.5,14.6) & 4.5(1.7,14.5) & 6.4 & 9.3 \\ \text { thiamine } & { }^{13} \mathrm{C}_{4} & 10 & 5.0(2.1,18.4) & 5.1(1.1,18.3) & 9 \\ \text { kynurenine } & { }^{2} \mathrm{H}_{4} & 200 & 4.5(1.8,13.6) & 4.9(1.6,14.8) & 5.7 & 6.5 \\ \text { tryptophan } & { }^{2} \mathrm{H}_{5} & 2000 & 4.6(1.2,15.1) & 4.3(2.0,18.9) & 6.2 & 6.5 \\ \text { anthranilic acid } & { }^{2} \mathrm{H}_{4} & 25 & 6.1(2.3,33.1) & 4.4(1.4,18.1) & 6.2\end{array}$

${ }^{a}$ Number and type of stable isotope substitutions for internal standard. ${ }^{b}$ concentration of internal standard. ${ }^{c}$ median (range) across 60 runs. Abbreviations IS, internal standard. 
Table S4. CVs for analytes and internal standards based on uncorrected peak areas on platform LC3

\begin{tabular}{|c|c|c|c|c|c|c|}
\hline \multirow[b]{3}{*}{ Analyte } & \multirow[b]{3}{*}{$I^{a}$} & \multirow[b]{3}{*}{ conc. $(\mathrm{nM})^{\mathrm{b}}$} & \multicolumn{4}{|c|}{ CV (\%) } \\
\hline & & & \multicolumn{2}{|c|}{ within-run ${ }^{c}$} & \multicolumn{2}{|c|}{ between-run } \\
\hline & & & analyte & internal standard & analyte & internal standard \\
\hline 25-hydroxy vitamin D3 & ${ }^{2} \mathrm{H}_{6}$ & 112 & $13.6(6.4,51)$ & $15.0(5.5,55)$ & 19.0 & 18.6 \\
\hline all-trans-retinol & ${ }^{2} \mathrm{H}_{6}$ & 1850 & $15.4(6.7,70)$ & $15.9(6.8,69)$ & 18.6 & 18.7 \\
\hline 25-hydroxy vitamin D2 & ${ }^{2} \mathrm{H}_{6}$ & 147 & $14.6(5.3,50)$ & $14.8(6.0,56)$ & 17.5 & 18.3 \\
\hline Y-tocopherol & ${ }^{2} \mathrm{H}_{9}$ & 4500 & $15.2(5.2,141)$ & $15.2(6.1,137)$ & 24.0 & 24.1 \\
\hline a-tocopherol & ${ }^{2} \mathrm{H}_{9}$ & 21000 & $15.5(6.4,136)$ & $14.8(8.3,128)$ & 26.1 & 25.2 \\
\hline phylloquinone & ${ }^{2} \mathrm{H}_{4}$ & 3.6 & $18.4(6.9,136)$ & $18.7(7.2,153)$ & 31.2 & 33.7 \\
\hline
\end{tabular}

${ }^{a}$ Number and type of stable isotope substitutions for internal standard. ${ }^{b}$ concentration of internal standard. ${ }^{c}$ median (range) across 60 runs. Abbreviations IS, internal standard. 
Table S5. CVs for matching and nonmatching A/IS on platform GC ${ }^{a}$

\begin{tabular}{|c|c|c|c|c|c|c|c|}
\hline \multirow[b]{3}{*}{ Analyte } & \multicolumn{5}{|c|}{ matching $\mathrm{A} / \mathrm{IS}$} & \multicolumn{2}{|c|}{ nonmatching $\mathrm{A} / \mathrm{IS}$} \\
\hline & \multirow{2}{*}{$\frac{\text { within-run }}{\mathrm{A} \|^{\mathrm{b}}}$} & \multicolumn{6}{|c|}{ between-run } \\
\hline & & S1 & S2 & S3 & All & $A l^{c}$ & CV difference ${ }^{d}$ \\
\hline methylmalonic acid & $2.4(1.6,4.5)$ & 2.6 & 1.7 & 3.1 & 2.8 & $9.7(5.3,19.8)$ & $6.8(2.5,17.0)$ \\
\hline 2-hydroxybutyrate & $1.7(0.9,3.9)$ & 2.1 & 1.9 & 1.8 & 2.3 & $11.0(6.0,17.7)$ & $8.8(3.7,15.4)$ \\
\hline 3-hydroxybutyrate & $1.9(0.7,8.8)$ & 2.5 & 2.2 & 3.8 & 3.4 & $11.8(3.7,17.7)$ & $8.4(0.3,14.4)$ \\
\hline alanine & $1.2(0.7,2.3)$ & 1.4 & 1 & 1.2 & 1.4 & $10.5(8.0,21)$ & $9.0(6.5,19.4)$ \\
\hline 3-hydroxyisobutyrate & $2.4(1.3,5.8)$ & 2.6 & 2.3 & 2.6 & 2.9 & $11.6(2.7,18.1)$ & $8.7(-0.3,15.2)$ \\
\hline glycine & $1.3(0.6,3.6)$ & 1.7 & 1.2 & 1.5 & 1.7 & $10.7(5.0,18.1)$ & $8.9(3.3,16.3)$ \\
\hline sarcosine & $1.3(0.6,4.1)$ & 1.5 & 1.3 & 1.5 & 1.6 & $9.2(5.4,19.9)$ & $7.6(3.7,18.3)$ \\
\hline valine & $2.2(0.8,5.6)$ & 2.5 & 2.5 & 2.7 & 2.7 & $12.6(6.8,18.9)$ & $9.9(4.1,16.2)$ \\
\hline leucine & $3.4(1.4,7.0)$ & 3.6 & 3.8 & 4 & 4 & $11.9(7.3,19.3)$ & $7.9(3.3,15.3)$ \\
\hline isoleucine & $1.5(0.5,3.5)$ & 1.8 & 1.4 & 1.6 & 1.8 & $10.4(4.9,19.2)$ & $8.5(3.1,17.3)$ \\
\hline a-Ketoglutarate & $2.3(1.1,4.9)$ & 2.5 & 1.9 & 3.1 & 3.6 & $10.8(7.0,20.2)$ & $7.2(3.5,16.6)$ \\
\hline threonine & $2.4(1.1,6.3)$ & 3.0 & 2.9 & 2.8 & 3.3 & $10.9(8.3,22.0)$ & $7.7(5.1,18.3)$ \\
\hline proline & $2.8(1.1,8.4)$ & 3.5 & 3 & 3.5 & 3.7 & $11.3(5.7,18.5)$ & $7.6(2.0,14.4)$ \\
\hline glutamine & $2.1(1.1,4.9)$ & 2.3 & 2.5 & 2 & 2.7 & $13.3(8.0,24)$ & $10.6(5.2,21)$ \\
\hline serine & $1.3(0.7,3.5)$ & 1.7 & 1.2 & 1.2 & 1.7 & $8.3(5.2,19.9)$ & $6.6(3.5,18.1)$ \\
\hline methionine & $2.2(0.7,6.6)$ & 2.7 & 2.2 & 2.1 & 4.1 & $8.4(6.3,17.7)$ & $4.4(2.3,13.6)$ \\
\hline
\end{tabular}




\begin{tabular}{|c|c|c|c|c|c|c|c|}
\hline glutamic acid & $1.7(0.9,3.4)$ & 1.9 & 1.8 & 1.5 & 2.7 & $10.7(7.4,21)$ & $8.0(4.7,18.3)$ \\
\hline phenylalanine & $1.5(0.8,7.0)$ & 2.1 & 2.4 & 2.5 & 3 & $11.0(5.7,18.7)$ & $8.1(2.8,15.7)$ \\
\hline homocysteine & $1.4(0.7,3.3)$ & 1.3 & 1.8 & 1.6 & 2.7 & $9.3(5.6,19.4)$ & $6.7(3.0,16.7)$ \\
\hline ornithine & $2.2(1.2,4.9)$ & 2.3 & 2.3 & 2.2 & 2.7 & $11.2(7.3,22)$ & $8.5(4.6,18.8)$ \\
\hline lysine & $1.4(0.4,3.7)$ & 1.6 & 1.5 & 1.5 & 1.9 & $8.4(5.4,20)$ & $6.5(3.5,18.4)$ \\
\hline tryptophan & $1.6(0.5,5.7)$ & 2.3 & 2.3 & 2.7 & 2.6 & $12.6(8.4,23)$ & $10.0(5.8,21)$ \\
\hline cystathionine & $2.3(1.2,4.6)$ & 2.9 & 1.8 & 2.1 & 2.9 & $15.7(9.0,25)$ & $12.8(6.1,22)$ \\
\hline kynurenine & $1.9(1.0,3.5)$ & 1.9 & 1.9 & 2.2 & 2.3 & $18.1(8.7,26)$ & $15.8(6.4,24)$ \\
\hline
\end{tabular}

${ }^{a}$ Numbers are CV (\%) based on all data (All) or specific source as indicated. ${ }^{b}$ Median (range) across 60 sets. ${ }^{c}$ Median (range) across all nonmatching A/IS. 'Median (range) difference nonmatching A/IS vs. matching A/IS across all nonmatching A/IS. Abbreviations A, analyte, IS, internal standard. S1, pooled EDTA plasma. S2, pooled serum spiked for most analytes. S3, pooled serum. 
Table S6. CVs for matching and nonmatching A/IS on platform $\mathrm{LC}^{\mathrm{a}}$

matching $A / I S$

nonmatching $A / I S$

\begin{tabular}{|c|c|c|c|c|c|c|c|}
\hline \multirow[b]{3}{*}{ Analyte } & & \\
\hline & \multirow{2}{*}{$\begin{array}{c}\text { within-run } \\
\mathrm{All}^{\mathrm{b}}\end{array}$} & \multicolumn{6}{|c|}{ between-run } \\
\hline & & S1 & S2 & S3 & All & $A l^{c}$ & CV difference \\
\hline histidine & $3.5(1.9,5.8)$ & 3.8 & 3.3 & 3.2 & 3.8 & $12.5(4.9,27)$ & $4.4(2.9,5.9)$ \\
\hline arginine & $2.6(1.5,4.4)$ & 2.9 & 2.3 & 2.2 & 3.0 & $15.1(5.4,31)$ & $2.6(1.5,4.1)$ \\
\hline dimethylglycine & $5.5(3.8,7.7)$ & 6.0 & 4.5 & 4.7 & 5.9 & $19.6(10.3,31)$ & $4.7(3.9,7.6)$ \\
\hline 1-methylhistidine & $3.7(2.5,5.7)$ & 4.0 & 3.3 & 3.2 & 3.9 & $9.1(5.1,20)$ & $2.9(1.4,5.1)$ \\
\hline methionine sulfoxide & $7.1(5.3,11.4)$ & 6.9 & 6.0 & 10.5 & 7.9 & $12.1(9.2,23)$ & $0.9(-0.2,2.6)$ \\
\hline trimethyllysine & $4.0(2.6,6.8)$ & 4.5 & 3.5 & 3.8 & 4.4 & $9.9(4.8,20)$ & $0.3(-1.1,2.7)$ \\
\hline creatine & $3.9(2.7,5.1)$ & 4.1 & 3.3 & 3.2 & 4.0 & $9.3(5.5,21)$ & $3.5(2.6 .6 .0)$ \\
\hline betaine & $3.8(2.2,9.4)$ & 4.2 & 4.5 & 3.6 & 4.5 & $11.8(5.2,24)$ & $3.5(2.6,6.0)$ \\
\hline homoarginine & $5.8(3.1,8.6)$ & 5.9 & 6.1 & 5.9 & 6.0 & $11.0(8.0,21)$ & $3.6(0.4,7.3)$ \\
\hline trimethylamine $\mathrm{N}$-oxide & $4.0(2.9,5.2)$ & 4.4 & 2.9 & 3.4 & 4.1 & $9.9(6.0,21)$ & $3.3(1.5,5.9)$ \\
\hline 3-methylhistidine & $4.4(2.9,7.0)$ & 4.8 & 3.9 & 3.9 & 4.9 & $11.5(8.0,23)$ & $1.7(0.1,3.6)$ \\
\hline choline & $2.8(1.7,5.4)$ & 3.1 & 2.6 & 2.6 & 3.2 & $11.0(5.3,23)$ & $2.7(0.5,4.4)$ \\
\hline symmetric dimethylarginine & $4.8(3.1,6.8)$ & 4.9 & 5.1 & 4.6 & 5.2 & $13.9(8.3,24$ & $3.9(2.4,5.8)$ \\
\hline asymmetric dimethylarginine & $5.2(3.7,8.0)$ & 5.7 & 4.3 & 4.6 & 5.5 & $11.3(9.6,21)$ & $2.1(-0.5,3.3)$ \\
\hline methionine & $4.1(2.8,7.0)$ & 4.4 & 4.4 & 3.2 & 4.5 & $11.4(7.2,24)$ & $3.0(0.5,4.6)$ \\
\hline creatinine & $2.6(1.2,5.3)$ & 2.9 & 2.6 & 2.5 & 3.1 & $9.0(4.3,22)$ & $5.9(1.2,18.7$ \\
\hline
\end{tabular}


${ }^{a}$ Numbers are CV (\%) based on all data (All) or specific source as indicated. ${ }^{b}$ Median (range) across 60 sets. ${ }^{c}$ Median (range) across all nonmatching A/IS. ${ }^{d}$ Median (range) difference nonmatching A/IS vs. matching A/IS across all nonmatching A/IS. Abbreviations A, analyte, IS, internal standard. S1, pooled EDTA plasma. S2, pooled serum spiked for most analytes. S3, pooled serum. 
Table S7. CVs for matching and nonmatching A/IS on platform LC2 ${ }^{\mathrm{a}}$

matching $A / I S$

nonmatching $\mathrm{A} / \mathrm{IS}$

\begin{tabular}{|c|c|c|c|c|c|c|c|}
\hline \multirow[b]{3}{*}{ Analyte } & \multicolumn{5}{|c|}{ matchıng A/IS } & \multicolumn{2}{|c|}{ nonmatching A/IS } \\
\hline & \multirow{2}{*}{$\begin{array}{c}\text { within-run } \\
\mathrm{All}^{\mathrm{b}}\end{array}$} & \multicolumn{6}{|c|}{ between-run } \\
\hline & & $\mathrm{S} 1$ & $\mathrm{~S} 2$ & S3 & All & $A l^{c}$ & CV difference ${ }^{d}$ \\
\hline neopterin & $5.3(2.8,10.7)$ & 5.7 & 4.3 & 6.5 & 6.6 & $9.2(8.1,10.7)$ & $2.6(1.5,4.1)$ \\
\hline cystathionine & $3.4(1.4,8.5)$ & 4.1 & 3.6 & 4.1 & 4.5 & $9.3(8.4,12.1)$ & $4.7(3.9,7.6)$ \\
\hline thiamine monophosphate & $7.1(2.8,29)$ & 8.5 & 8.9 & 7.8 & 11.6 & $12.5(11.4,14.2)$ & $0.9(-0.2,2.6)$ \\
\hline trimethylamine $\mathrm{N}$-oxide & $4.2(2.3,21)$ & 5.7 & 4.9 & 5.8 & 6.9 & $7.2(5.8,9.6)$ & $0.3(-1.1,2.7)$ \\
\hline n-methylnicotinamide & $4.0(1.6,9.4)$ & 4.6 & 4.3 & 3.7 & 5.5 & $9.0(5.5,12.5)$ & $3.5(2.6 .6 .0)$ \\
\hline 4-pyridoxic acid & $3.6(1.4,8.7)$ & 4.0 & 4.4 & 4.0 & 5.8 & $7.5(6.0,9.5)$ & $1.7(0.1,3.6)$ \\
\hline pyridoxal & $3.5(2.0,7.3)$ & 3.8 & 3.9 & 3.7 & 5.1 & $7.8(5.6,9.4)$ & $2.7(0.5,4.4)$ \\
\hline flavin mononucleotide & $5.7(3.0,18.3)$ & 6.4 & 7.4 & 7.3 & 8.1 & $12.0(10.5,14.0)$ & $3.9(2.4,5.8)$ \\
\hline pyridoxin & $4.5(2.2,7.6)$ & 4.8 & 4.4 & 4.7 & 5.2 & $7.3(4.7,8.5)$ & $2.1(-0.5,3.3)$ \\
\hline cotinine & $3.0(1.2,7.4)$ & 3.6 & 3.4 & 3.8 & 4.2 & $7.2(4.8,8.8)$ & $3.0(0.5,4.6)$ \\
\hline 3-hydroxykynurenine & $3.2(1.3,7.8)$ & 3.9 & 4.3 & 3.4 & 4.5 & $7.8(6.3,9.7)$ & $3.4(1.8,5.2)$ \\
\hline
\end{tabular}




\begin{tabular}{|c|c|c|c|c|c|c|c|}
\hline 3-hydroxyanthranilic acid & $4.6(1.9,13.2)$ & 5.9 & 5.1 & 5.1 & 6.0 & $8.8(6.8,10.2)$ & $2.8(0.8,4.1)$ \\
\hline xanthurenic acid & $4.8(1.7,10.1)$ & 5.4 & 4.8 & 4.7 & 5.7 & $8.5(6.8,11.3)$ & $2.8(1.1,5.6)$ \\
\hline riboflavin & $6.0(2.2,11.5)$ & 6.8 & 5.8 & 5.6 & 7.1 & $8.3(7.1,10.9)$ & $1.2(0.0,3.8)$ \\
\hline kynurenic acid & $4.4(1.8,13.9)$ & 5.1 & 4.6 & 5.5 & 5.4 & $7.4(5.8,9.8)$ & $2.0(0.4,4.4)$ \\
\hline thiamine & $3.5(1.8,9.9)$ & 5.3 & 3.5 & 3.9 & 4.9 & $9.6(8.0,11.8)$ & $4.7(3.1,6.9)$ \\
\hline kynurenine & $3.6(1.9,13.6)$ & 4.9 & 4.2 & 4.9 & 5.1 & $7.1(5.1,8.7)$ & $2.0(-0.1,3.6)$ \\
\hline tryptophan & $3.1(1.5,6.8)$ & 3.6 & 3.7 & 3.2 & 3.9 & $7.4(5.1,9.0)$ & $3.5(1.2,5.2)$ \\
\hline anthranilic acid & $7.4(2.5,24)$ & 5.5 & 11.8 & 9.6 & 9.5 & $11.4(10.1,13.3)$ & $1.9(0.6,3.8)$ \\
\hline
\end{tabular}

${ }^{a}$ Numbers are CV (\%) based on all data (All) or specific source as indicated. ${ }^{b}$ Median (range) across 60 sets. ${ }^{\mathrm{C}}$ Median (range) across all nonmatching A/IS. 'Median (range) difference nonmatching A/IS vs. matching A/IS across all nonmatching A/IS. Abbreviations A, analyte, IS, internal standard. S1, pooled EDTA plasma. S2, pooled serum spiked for most analytes. S3, pooled serum. Table S4a. Platform LC3 
Table S8. CVs for matching and nonmatching A/IS on platform LC3 ${ }^{a}$

matching $A / I S$

nonmatching $\mathrm{A} / \mathrm{IS}$

\begin{tabular}{|c|c|c|c|c|c|c|c|}
\hline \multirow[b]{3}{*}{ Analyte } & & \\
\hline & \multirow{2}{*}{$\begin{array}{c}\text { within-run } \\
\mathrm{A} \|^{\mathrm{b}}\end{array}$} & \multicolumn{6}{|c|}{ between-run } \\
\hline & & S1 & S2 & S3 & All & All ${ }^{\mathrm{C}}$ & CV difference ${ }^{d}$ \\
\hline 25-hydroxy vitamin D3 & $5.1(3.2,13.5)$ & 6.3 & 5.1 & 5.4 & 6.1 & $14.4(8.8,25.3)$ & $8.3(2.7,19.2)$ \\
\hline all-trans-retinol & $2.9(1.6,7.2)$ & 3.8 & 3.0 & 2.6 & 3.6 & $14.8(7.6,26.6)$ & $11.3(4.1,23.0)$ \\
\hline 25-hydroxy vitamin D2 & $6.6(1.9,22)$ & 7.3 & 7.2 & 8.1 & 8.1 & $16.1(9.9,28.3))$ & $8.0(1.8,20.2)$ \\
\hline Y-tocopherol & $3.7(1.4,10.8)$ & 4.7 & 4.5 & 4.1 & 5.0 & $15.6(9.5,21.3)$ & $10.6(4.6,16.3)$ \\
\hline a-tocopherol & $4.3(2.0,13.4)$ & 5.3 & 5.8 & 4.6 & 5.8 & $18.0(9.7,18.4)$ & $12.2(3.9,12.6)$ \\
\hline phylloquinone & $4.8(2.1,14.8)$ & 5.9 & 6.8 & 6.6 & 7.4 & $24.1(18.9,26.7)$ & $16.7(11.5,19.4)$ \\
\hline
\end{tabular}

${ }^{a}$ Numbers are CV (\%) based on all data (All) or specific source as indicated. ${ }^{b}$ Median (range) across 60 sets. ${ }^{c}$ Median (range) across all nonmatching A/IS. ${ }^{d}$ Median (range) difference nonmatching A/IS vs. matching A/IS across all nonmatching A/IS. Abbreviations A, analyte, IS, internal standard. S1, pooled EDTA plasma. S2, pooled serum spiked for most analytes. S3, pooled serum. 